\title{
Underlying cardiopulmonary conditions as a risk factor for influenza and respiratory syncytial virus infection among community-dwelling adults aged [?]65 years in Thailand: Findings from a two-year prospective cohort study
}

Prabda Praphasiri ${ }^{1}$, Manash Shrestha ${ }^{2}$, Jayanton Patumanond ${ }^{3}$, Sutthichai Nakphook ${ }^{4}$, Sutthinan Chawalchitiporn ${ }^{5}$, Darunee Ditsungnoen ${ }^{6}$, Fatimah Dawood ${ }^{7}$, Joshua Mott ${ }^{8}$, and Kriengkrai Prasert ${ }^{5}$

${ }^{1}$ Thailand MOPH-US CDC Collaboration

${ }^{2}$ Mahidol University

${ }^{3}$ Chiang Mai University

${ }^{4}$ Institute of Preventive Medicine, Department of Disease Control, Ministry of Public

Health

${ }^{5}$ Nakhon Phanom Provincial Hospital

${ }^{6}$ Thailand Ministry of Public Health - U.S. Centers for Disease Control and Prevention

Collaboration

${ }^{7}$ Centres for Disease Control and Prevention

${ }^{8} \mathrm{CDC}$

February 5, 2021

\begin{abstract}
Background: Adults with cardiopulmonary conditions are at increased risk for complications from influenza and respiratory syncytial virus (RSV) infection, but few data are available from middle-income countries. Methods: Using data from a prospective cohort study of influenza vaccine effectiveness among community-dwelling Thai adults aged [?]65 years, we estimated and compared the incidence of influenza and RSV in those with and without cardiopulmonary conditions. During May 2015-May 2017, older adults in a rural province in Thailand were followed weekly with year-round surveillance for acute respiratory illness (ARI), defined broadly as new onset or worsening of cough with or without fever, and hospitalized ARI. When ill, nasal selfswabs and/or nasopharyngeal swabs were collected for reverse-transcription polymerase chain reaction testing. We used Poisson regression to calculate incidence rate ratios (IRR), adjusting for age, sex, past healthcare-seeking behavior, weekly influenza activity, and influenza vaccination. Results: Overall, 3,220 adults with a median age of 71 years (IQR 68-76) were enrolled; $1,324(41.1 \%)$ were male; and $313(9.7 \%)$ had [?]1 underlying cardio-pulmonary condition, most commonly chronic obstructive pulmonary disease $(131 ; 41.2 \%)$ or asthma $(73 ; 23.3 \%)$. Compared to those without, participants with cardiopulmonary conditions had higher incidences of ARI, influenza, and RSV (Adjusted IRR: 1.83, 95\% CI 1.63-2.05; 1.84, 95\% CI 1.05-3.23; 2.02, 95\% CI 1.10-3.72, respectively). Conclusion: Our findings show that older adults in rural Thailand with cardiopulmonary conditions have increased rates of ARI, influenza, and RSV infections, and support efforts to ensure this population has access to influenza vaccines and other respiratory illness prevention measures.
\end{abstract}

\section{INTRODUCTION}

Influenza and respiratory syncytial virus (RSV) are respiratory pathogens that cause a substantial disease 
burden among adults aged 65 years and above [1]. Global estimates suggest that around two-thirds of all seasonal influenza-related mortality occurs among older adults with more than 260,000 deaths annually [2]. Data from developed countries show that respiratory illnesses due to RSV among older adults may exceed one million episodes and cause around 14,000 in-hospital deaths every year [3]. Older adults with cardiopulmonary conditions are at increased risk for severe illness due to influenza and RSV infection [4, 5]. However, it is not fully established whether cardiopulmonary conditions present an increased risk for influenza and RSV infections, and there are little data on the burden of infection from these pathogens among persons with cardiopulmonary conditions in middle-income countries.

Thailand is an upper-middle-income country in tropical Southeast Asia, where recognition of influenza and RSV disease burden is growing. Since 2008, adults aged [?]65 years and those with chronic diseases have been recommended for annual influenza vaccination by the Thai Ministry of Public Health (MoPH) [6]. Nonetheless, influenza vaccine uptake among older adults is still less than 20\% (Bureau of General Communicable Diseases, Thailand MoPH). Studies among hospitalized patients suggest that the prevalence of RSV-associated respiratory illnesses in Thailand is around 2-9\% among older adults [7-11]. However, reliable estimates of the infection rates in the community remain limited. Using data from a longitudinal prospective cohort study of influenza vaccine effectiveness among rural community-dwelling Thai adults aged [?]65 years [12], we compared the incidence of influenza and RSV in those with and without cardiopulmonary conditions.

\section{METHODS}

\section{Study design}

Details of this study have been described previously [12]. In brief, a prospective cohort of 3500 adults aged [?]65 years was recruited by systematic random sampling in two districts of Nakhon Phanom province, Thailand from May 2015 and followed for two years until May 2017. Prior to the start of the study, a targeted campaign was organized in the study sites by the Thai MoPH to enhance influenza vaccine uptake among older adults through mobile clinics and increased vaccine supply [13]. Individuals were not eligible to participate in the cohort if they resided in a long term care facility, did not reside in the community for at least a year, were unable to communicate with study staff, were unable/unwilling to provide nasal self-swabs, or had an acute medical condition or tumor in or near the nose that would preclude nasal swab collection. Trained health volunteers collected baseline data and followed the participants with weekly telephone surveillance for acute respiratory illness (ARI). Participants were asked to self-collect anterior nasal swabs with each ARI episode as previously validated [14]. For participants who visited health centers/hospitals for their illnesses, nasopharyngeal swabs were collected by research nurses on site. The number of hospital visits of the participants (for ARI or other reasons) during the study years were obtained from the district and provincial hospital records.

\section{Definitions}

Acute respiratory illness (ARI) was defined as a new onset of cough or worsening of a chronic cough. Severe ARI (SARI) was defined as an illness requiring hospitalization with measured axillary temperature [?] $38^{\circ} \mathrm{C}$ plus a new onset of a cough, worsening of a chronic cough, or difficulty in breathing. Cardiopulmonary condition was defined as the presence of any known physician-diagnosed chronic cardiovascular conditions (such as coronary artery diseases, cardiac arrhythmias, valvular heart diseases, cardiomyopathy) and/or any chronic lung diseases (such as chronic obstructive pulmonary disease (COPD), asthma, chronic bronchitis, pulmonary tuberculosis, emphysema, and lung fibrosis).

\section{Specimen collection, storage, laboratory testing}

All respiratory specimens (including participant-collected and nurse-collected swabs) were transported to refrigerators at sub-district health centers within 24 hours of collection, kept between $2-8{ }^{\circ} \mathrm{C}$ before being frozen in liquid nitrogen tanks below $-70{ }^{\circ} \mathrm{C}$, and sent weekly to the Thai National Institute of Health (NIH) 
in Nonthaburi. Respiratory specimens were tested for influenza viruses and RSV with real-time reversetranscription polymerase chain reaction test (rRT-PCR) according to CDC/WHO protocols $[15,16]$.

\section{Data analysis}

We calculated the incidence per 1000 person-years of laboratory-confirmed influenza and RSV-associated ARI and SARI by dividing the total episodes by total person-time under observation. Weeks of intermittent loss to follow up (due to missed swab collection and/or missed surveillance contacts) were excluded from the person-time denominator when calculating the incidence rates. Multi-variable Poisson regression was used to calculate incidence rate ratios (IRR) between participants with and without any underlying cardiopulmonary conditions, adjusting for age, sex, influenza vaccination status (participants receiving influenza vaccine in at least one of the two study years were considered as vaccinated), weekly influenza activity (influenza infections detected by weekly NIH surveillance, categorized into quartiles), and number of hospital visits during the past two years as a proxy for healthcare seeking behavior. All data analyses were conducted using STATA software version 14.2 (StataCorp LP, College Station, TX, USA).

\section{Ethical considerations}

Written informed consent was obtained from all participants and the study was approved by the Ethical Review Committee, Department of Disease Control, MoPH (Nonthaburi, Thailand). The US Centers for Disease Control and Prevention (Atlanta, Georgia) relied on this committee for ethical approval.

\section{RESULTS}

\section{Participant Characteristics}

Of 3,500 participants screened, 3,287 were eligible, and 3,220 adults were enrolled in the study. The participants had a median age of 71 years (interquartile range [IQR] 68-76) and 1,324 (41\%) were male (Table 1). The proportion of cohort participants that were vaccinated for influenza was $52 \%(1,666 / 3,220)$ in 2015 and $48 \%(1,498 / 3,123)$ in 2016 . In total, $313(9.7 \%)$ had one or more underlying cardiopulmonary condition, most commonly COPD $(131 ; 41.2 \%)$, asthma $(73 ; 23.3 \%)$, or chronic bronchitis $(68 ; 21.7 \%)$ (Table 2 ). The participants with cardiopulmonary conditions were more likely to be older, male, have more other co-morbidities, and have a higher number of hospital visits than those without cardiopulmonary conditions (Table 1).

The participants were followed-up for a total 316,690 person-weeks (29,005 person-weeks for participants with cardiopulmonary conditions and 287,685 person-weeks for participants without cardiopulmonary conditions). During the two-year follow-up, two participants withdrew and 200 (6.2\%) died. Overall, 2365 ARI/SARI episodes (2296 ARI and $69 \mathrm{SARI})$ were reported in the cohort of which $2362(99.8 \%)$ respiratory swabs were collected (1969 nasal self-swabs and 393 nasopharyngeal swabs by research nurses).

\section{Incidence of influenza and RSV}

Influenza $\mathrm{A}(\mathrm{H} 3 \mathrm{~N} 2)$ was the predominant circulating influenza virus in both years. The median time from symptom onset to collection of nasal swabs was 1 day (IQR 1-2) and nasopharyngeal swabs was 2 days (IQR 1-3), which were not different among participants with or without cardiopulmonary conditions (p-values 0.589 and 0.746 , respectively). Overall, $105(3.3 \%)$ cohort participants had an influenza virus infection (influenza $\mathrm{A}(\mathrm{H} 1 \mathrm{~N} 1)=17 ; \mathrm{A}(\mathrm{H} 3 \mathrm{~N} 2)=74 ; \mathrm{B}=14)$ and $81(2.5 \%)$ had an RSV infection. Laboratory-confirmed influenza infections were detected most in the first year in September (18.4\%), followed by October in the second year (12.2\%); most RSV infections were detected during August to October in the second year, with the peak in September at $22.0 \%$ (Figure 1).

Participants with cardiopulmonary conditions had a higher incidence of ARI (645/1000 person-years vs $350 / 1000$ person-years, $\mathrm{p}<0.001)$ and SARI $(87.8 / 1000$ vs $3.6 / 1000, \mathrm{p}<0.001)$ than those without. Adjusted IRR for laboratory-confirmed RSV, all influenza, influenza A, and influenza B viruses were 2.02 (95\% confidence interval [CI] 1.10-3.72), 1.84 (95\% CI 1.05-3.23), 1.83 (95\% CI 1.00-3.34), and 1.96 (95\% CI 0.42-9.21), respectively (Table 3 ). 


\section{DISCUSSION}

Among a two-year prospective cohort study of $>3,000$ community dwelling adults in Thailand, around $10 \%$ of participants had underlying cardiopulmonary conditions and cardiopulmonary conditions conferred increased rates of all-cause ARI, influenza A and RSV infections. These findings provide additional support for influenza vaccination recommendations for persons with underlying cardiopulmonary conditions and add to the limited body of evidence about influenza and RSV disease burden among older adults in middle-income countries.

The seasonality of influenza and RSV was similar to the pattern identified in past studies in which the infections peaked during the rainy months of June to October $[10,17,18]$. Incidences of influenza and RSV are higher in our study than previous studies $[7,8,10]$. This may be partly due to a more sensitive case definition of ARI. Since many older adults with influenza and RSV can present without fever, we used a definition of ARI without any body temperature criteria [12]. This might have resulted in the inclusion of more illness episodes with laboratory confirmation than in the past studies which required either fever or hypothermia $\left(<35.5^{\circ} \mathrm{C}\right)$ to trigger swab collection. Moreover, previous studies primarily assessed hospitalized patients and may not have fully accounted for non-medically attended cases in the community, even after adjustments in their analyses $[7,8]$. In our study, more than $80 \%$ of the respiratory specimens were nasal self-swabs collected by the participants at their homes and only a small proportion of ARI were severe enough to warrant hospitalization, signifying a majority of non-medically attended cases in the community.

Cardiopulmonary conditions are recognized risk factors for severe outcomes of influenza and RSV infections, especially among older adults $[4,5]$. Prior studies have demonstrated that a disproportionate number of hospitalizations for influenza and RSV occur in patients with cardiopulmonary conditions, particularly COPD $[9,10,18]$. Our findings suggest that these conditions may themselves be predisposing risk factors for acquisition of influenza and RSV infections in the community, possibly by affecting immunoregulatory functions in older adults [19]. Our findings are consistent with evidence from Western countries that documents higher detection of RSV among older adults with underlying cardiopulmonary diseases compared to those without these conditions $[19,20]$.

There are some limitations to our study. First, we ascertained cardiopulmonary conditions by participant report alone which may be susceptible to some recall and measurement bias. Second, we did not collect data about exposure to children, household crowding, socioeconomic status, and baseline influenza and RSV antibody titers which may be confounders in the relationship between cardiopulmonary conditions and acquisition/transmission of influenza virus and RSV infection [19]. Third, a previous analysis comparing participant-collected nasal swabs to nurse-collected nasopharyngeal swabs among older adults in Thailand found that participant-collected nasal swabs were only $78 \%$ sensitive for detection of influenza viruses [14]. Therefore, our calculation of influenza and RSV incidence may be underestimated. Nevertheless, the strengths of this research lie in the population-based multiple-year prospective cohort study design with a large sample size, use of a broad case definition of ARI, capture of non-medically attended ARI, active weekly follow-up of participants, and laboratory-confirmation of the infection outcomes.

In conclusion, incidences of influenza and RSV were high among community-dwelling older Thai adults aged $>65$ years in this study, and cardiopulmonary conditions conferred additional risk for acquisition of influenza and RSV infections. Despite efforts to enhance influenza vaccine uptake among this cohort as part of the study design, only half of participants received influenza vaccines during each of the study years. More efforts are needed to ensure adults aged $>65$ years, especially those with cardiopulmonary conditions, have access to influenza vaccines and other prevention measures as effective vaccines against RSV are not currently available.

\section{Notes}

Funding

This research was supported by US Centers for Disease Control and Prevention and awarded to the Ministry 
of Public Health, Thailand in cooperative agreement 5-U01GH000152.

Disclaimer

The findings and conclusions in this report are those of the authors and do not necessarily represent the views of the Centers for Disease Control and Prevention.

Conflict of interest

The authors declare that they have no personal or financial relationship that could lead to a conflict of interest.

\section{References :}

1. Elliot AJ, Fleming DM. Influenza and respiratory syncytial virus in the elderly. Expert Rev Vaccines 2008 ; 7(2): 249-58.

2. Paget J, Spreeuwenberg P, Charu V, et al. Global mortality associated with seasonal influenza epidemics: New burden estimates and predictors from the GLaMOR Project. J Glob Health 2019 ; 9(2): 020421.

3. Shi T, Denouel A, Tietjen AK, et al. Global Disease Burden Estimates of Respiratory Syncytial VirusAssociated Acute Respiratory Infection in Older Adults in 2015: A Systematic Review and Meta-Analysis. J Infect Dis 2020 ; 222(Supplement_7):S577-S583.

4. Grohskopf LA, Sokolow LZ, Broder KR, et al. Prevention and Control of Seasonal Influenza with Vaccines: Recommendations of the Advisory Committee on Immunization Practices - United States, 2017-18 Influenza Season. MMWR Recommendations and reports: MMWR Morb Mortal Wkly Rep2017 ; 66(2): 1-20.

5. Falsey AR, Walsh EE, Esser MT, Shoemaker K, Yu L, Griffin MP. Respiratory syncytial virus-associated illness in adults with advanced chronic obstructive pulmonary disease and/or congestive heart failure. J Med Virol 2019 ; 91(1): 65-71.

6. Owusu JT, Prapasiri P, Ditsungnoen D, et al. Seasonal influenza vaccine coverage among high-risk populations in Thailand, 2010-2012. Vaccine $2015 ; 33(5)$ : 742-7.

7. Fry AM, Chittaganpitch M, Baggett HC, et al. The Burden of Hospitalized Lower Respiratory Tract Infection due to Respiratory Syncytial Virus in Rural Thailand. PLOS ONE 2010 ; 5(11): e15098.

8. Olsen SJ, Thamthitiwat S, Chantra S, et al. Incidence of respiratory pathogens in persons hospitalized with pneumonia in two provinces in Thailand. Epidemiol Infect 2010 ; 138(12): 1811-22.

9. Hara K, Yahara K, Gotoh K, et al. Clinical Study Concerning the Relationship between CommunityAcquired Pneumonia and Viral Infection in Northern Thailand. Intern Med 2011 ; 50(9): 991-8.

10. Naorat S, Chittaganpitch M, Thamthitiwat S, et al. Hospitalizations for Acute Lower Respiratory Tract Infection Due to Respiratory Syncytial Virus in Thailand, 2008-2011. J Infect Dis 2013 ; 208(suppl_3): S238-S45.

11. Thongpan I, Suntronwong N, Vichaiwattana P, Wanlapakorn N, Vongpunsawad S, Poovorawan Y. Respiratory syncytial virus, human metapneumovirus, and influenza virus infection in Bangkok, 2016-2017. PeerJ 2019 ; 7: e6748-e.

12. Prasert K, Patumanond J, Praphasiri P, et al. Effectiveness of trivalent inactivated influenza vaccine among community-dwelling older adults in Thailand: A two-year prospective cohort study. Vaccine2019 ; 37(6): 783-91.

13. Praphasiri P, Ditsungnoen D, Sirilak S, et al. Predictors of seasonal influenza vaccination among older adults in Thailand. PLOS ONE2017 ; 12(11): e0188422. 
14. Goyal S, Prasert K, Praphasiri P, et al. The acceptability and validity of self-collected nasal swabs for detection of influenza virus infection among older adults in Thailand. Influenza Other Resp2017 ; 11(5): 412-7.

15. World Health Organization. WHO information for the molecular detection of influenza viruses. World Health Organization, Geneva, Switzerland http://www.who int/influenza/gisrs_laboratory/molecular_diagnosis/en 2017.

16. World Health Organization. CDC protocol of realtime RTPCR for swine influenza A (H1N1). CDC protocol of realtime RTPCR for swine influenza A (H1N1): World Health Organization (WHO), 2009 .

17. Chittaganpitch M, Waicharoen S, Yingyong T, et al. Viral etiologies of influenza-like illness and severe acute respiratory infections in Thailand. Influenza Other Resp 2018 ; 12(4): 482-9.

18. Chuaychoo B, Ngamwongwan S, Kaewnaphan B, et al. Clinical manifestations and outcomes of respiratory syncytial virus infection in adult hospitalized patients. J Clin Virol 2019 ; 117: 103-8.

19. Mehta J, Walsh EE, Mahadevia PJ, Falsey AR. Risk Factors for Respiratory Syncytial Virus Illness Among Patients with Chronic Obstructive Pulmonary Disease. COPD 2013 ; 10(3): 293-9.

20. Ivey KS, Edwards KM, Talbot HK. Respiratory Syncytial Virus and Associations With Cardiovascular Disease in Adults. J Am Coll Cardiol 2018 ; 71(14): 1574-83.

Table 1. Participant characteristics in a population-based cohort study, Nakhon Phanom Province, Thailand, May 2015 - May 2017

\begin{tabular}{|c|c|c|c|}
\hline Characteristics & Cohort participants & Cohort participants & $\mathrm{p}$-value \\
\hline & $\begin{array}{l}\text { Without } \\
\text { cardiopulmonary } \\
\text { conditions }(\mathrm{n}=2,907) \mathrm{n} \\
(\%)\end{array}$ & $\begin{array}{l}\text { With cardiopulmonary } \\
\text { conditions }(\mathrm{n}=313) \mathrm{n} \\
(\%)\end{array}$ & \\
\hline Total person-years & 5532 & 558 & \\
\hline$<75$ years & $1,925(66.2)$ & $190(60.7)$ & 0.052 \\
\hline [?]75 years & $982(33.8)$ & $123(39.3)$ & \\
\hline Male & $1,157(39.8)$ & $167(53.4)$ & $<0.001$ \\
\hline \multicolumn{4}{|l|}{$\begin{array}{l}\text { Influenza vaccination } \\
\text { uptake }\end{array}$} \\
\hline $2015-2016$ & $1,497(51.5)$ & $169(54.0)$ & 0.405 \\
\hline $2016-2017$ & $1,347(47.5)$ & $151(53.0)$ & 0.082 \\
\hline \multicolumn{4}{|l|}{ Co-morbidity } \\
\hline Yes & $1,163(40.0)$ & $156(49.8)$ & $<0.001$ \\
\hline Hypertension & $670(23.1)$ & $85(27.2)$ & 0.107 \\
\hline Diabetes & $543(18.7)$ & $62(19.8)$ & 0.648 \\
\hline Neuromuscular diseases & $83(2.9)$ & $14(4.5)$ & 0.117 \\
\hline Chronic kidney diseases & $79(2.7)$ & $11(3.5)$ & 0.370 \\
\hline Cerebrovascular diseases & $74(2.6)$ & $11(3.5)$ & 0.350 \\
\hline Chronic liver diseases & $13(0.5)$ & $3(1.0)$ & 0.199 \\
\hline Thalassemia & $4(0.1)$ & $6(1.9)$ & $<0.001$ \\
\hline Cancer & $81(2.8)$ & $17(5.4)$ & 0.015 \\
\hline Chemotherapy & $10(0.3)$ & $2(0.6)$ & 0.328 \\
\hline
\end{tabular}




\begin{tabular}{llll}
\hline Characteristics & Cohort participants & Cohort participants & p-value \\
\hline $\begin{array}{l}\text { Mean number of } \\
\text { hospital visits (SD) }\end{array}$ & $2.8(1.6)$ & $3.5(1.1)$ & $<0.001$ \\
\hline
\end{tabular}

Abbreviations: IQR, interquartile range; $n$, number.

Table 2. Cardiopulmonary conditions among participants in a population-based cohort study, Nakhon Phanom Province, Thailand, May 2015 - May 2017

\begin{tabular}{ll}
\hline Cardiopulmonary condition $^{\mathbf{a}}$ & Total=313 \\
\hline 1. Cardiovascular diseases & $\mathbf{n} \mathbf{( \% )}$ \\
Coronary artery diseases & $48(15.3)$ \\
Cardiac arrythmias & $27(8.6)$ \\
Heart valve diseases & $10(3.2)$ \\
Cardiomyopathy & $3(1.0)$ \\
2. Chronic lung diseases & $131(41.2)$ \\
Chronic obstructive pulmonary disease & $73(23.3)$ \\
Asthma & $68(21.7)$ \\
Chronic bronchitis & $39(12.5)$ \\
Pulmonary tuberculosis & $1(0.3)$ \\
Emphysema & $1(0.3)$ \\
Lung fibrosis &
\end{tabular}

${ }^{a}$ Each participant could have more than one cardiopulmonary condition

Table 3. Incidence rate ratio of Respiratory Syncytial Virus, Influenza A and B among participants with or without cardiopulmonary conditions

\begin{tabular}{|c|c|c|c|c|c|c|}
\hline & $\begin{array}{l}\text { Participants } \\
\text { without } \\
\text { CPC }\end{array}$ & $\begin{array}{l}\text { Participants } \\
\text { without } \\
\text { CPC }\end{array}$ & $\begin{array}{l}\text { Participants } \\
\text { with CPC }\end{array}$ & $\begin{array}{l}\text { Participants } \\
\text { with CPC }\end{array}$ & $\begin{array}{l}\text { Crude IRR } \\
(95 \% \text { CI })\end{array}$ & $\begin{array}{l}\text { Adjusted } \\
\text { IRR }^{d}(95 \% \\
\text { CI })\end{array}$ \\
\hline $\begin{array}{l}\text { No. of } \\
\text { person }\end{array}$ & 2,907 & 2,907 & 313 & 313 & & \\
\hline Person-years & 5,532 & 5,532 & 558 & 558 & & \\
\hline Endpoints & No. & $\begin{array}{l}\text { Rate }^{\mathrm{a}}(95 \% \\
\text { CI) }\end{array}$ & No. & $\begin{array}{l}\text { Rate }^{\mathrm{a}}(95 \% \\
\text { CI })\end{array}$ & & \\
\hline $\mathrm{ARI}^{\mathrm{b}}$ & 1,936 & $350(337,363)$ & 360 & $645(604,685)$ & $\begin{array}{l}1.84(1.64 \\
2.07)^{*}\end{array}$ & $\begin{array}{l}1.83(1.63, \\
2.05)^{*}\end{array}$ \\
\hline $\mathrm{SARI}^{\mathrm{C}}$ & 20 & $3.6(2.2,5.6)$ & 49 & $\begin{array}{l}87.8(65.7, \\
114.4)\end{array}$ & $\begin{array}{l}24.30(14.18 \\
43.16)^{*}\end{array}$ & $\begin{array}{l}24.67(14.11 \\
43.16)^{*}\end{array}$ \\
\hline RSV infection & 68 & $12.3(9.6,15.6)$ & 13 & $\begin{array}{l}23.2(12.5, \\
39.5)\end{array}$ & $\begin{array}{l}1.90(0.96 \\
3.46)^{*}\end{array}$ & $\begin{array}{l}2.02(1.10, \\
3.72)^{*}\end{array}$ \\
\hline Influenza & 90 & $\begin{array}{l}16.3(13.1, \\
20.0)\end{array}$ & 15 & $\begin{array}{l}26.9(15.1, \\
44.0)\end{array}$ & $\begin{array}{l}1.65 \\
(0.89,2.87)\end{array}$ & $\begin{array}{l}1.84(1.05 \\
3.23)^{*}\end{array}$ \\
\hline Influenza A & 78 & $14.1(9.9,16.9)$ & 13 & $\begin{array}{l}23.3(12.5, \\
39.5)\end{array}$ & $\begin{array}{l}1.65(0.84, \\
2.99)\end{array}$ & $\begin{array}{l}1.83(1.00, \\
3.34)^{*}\end{array}$ \\
\hline Influenza B & 12 & $2.2(1.1,3.9)$ & 2 & $3.6(0.4,12.9)$ & $\begin{array}{l}1.65(0.18, \\
7.43)\end{array}$ & $\begin{array}{l}1.96(0.42, \\
9.21)\end{array}$ \\
\hline
\end{tabular}


Abbreviations: ARI, acute respiratory illness; CI, confidence interval; CPC, cardiopulmonary conditions; IRR, incidence rate ratio; No., Number; RSV, Respiratory syncytial virus; SARI, severe acute respiratory illness.

${ }^{a}$ Incidence rate / 1,000 person-years.

${ }^{b}$ ARI defined as a new onset of cough or worsening of chronic cough, with or without fever.

${ }^{c}$ SARI defined as new onset of cough, worsening of chronic cough or difficulty breathing with a fever [?] $38.0^{\circ} \mathrm{C}$ that required hospitalization.

${ }^{d}$ Poisson regression analysis adjusted for age, sex, influenza vaccination, weekly influenza activity, and number of hospital visits.

*Statistically significant at p-value $<0.05$.

\section{Figure legend:}

Figure 1. Number of laboratory-confirmed influenza and RSV infection and proportion of positive cases by month among older adults aged [?]65 years in population-based cohort study, Nakhon Phanom province, Thailand, May 2015 - May 2017

\section{Hosted file}

figure word.pdf available at https://authorea.com/users/393909/articles/507417-underlyingcardiopulmonary-conditions-as-a-risk-factor-for-influenza-and-respiratory-syncytialvirus-infection-among-community-dwelling-adults-aged-65-years-in-thailand-findings-froma-two-year-prospective-cohort-study 